\title{
A STUDY ON SUBCLINICAL KETOSIS IN HOLSTEIN CATTLE OF TORBAT-HEYDARIEH (KHORASAN PROVINCE)
}

\author{
Pourjafar, M. and Heidari, $M$. \\ School of Veterinary Medicine, Shahrekord University, Shahrekord, IRAN. \\ Fax: 0098-381-4424412 \\ E-mail:dmp4m@yahoo.com
}

\begin{abstract}
Ketosis is a production disease that is common in heavily producing cows in the post-calving period. Various studies have shown that subclinical ketosis is common in high-producing cows, 2-7 weeks postpartum (1). Clinical and subclinical ketosis is one of the major causes of loss to the dairy farmers and the main economic loss is due to the loss of production while the disease is present and failure to return to full production after recovery. In addition, subclinical ketosis increases the risk of delayed estrus, cystic ovarian disease, mastitis and increased intercalving interval.

This study was designed to determine the prevalence of subclinical ketosis and its relation to postparturient diseases (mastitis, metritis and retained placenta), age, and milk yield. Milk samples ( $\mathrm{n}=$ 511) from 511 dairy Iranian Holstein cows in 12 Torbat-Heydarieh (Khorasan province) dairy herds were studied from March 1998 to May 1999. Cows were sampled randomly in 2 to 10 weeks after parturition. Milk samples were stored in centrifuged tube and immediately examined by Rothera test. Chi-square test was used for statistical evaluation at the level of $\mathrm{P}<0.05$.

Results indicated that the cumulative frequency of subclinical ketosis was 38\%. No significant relation was observed between metritis, retained placenta, milk yield and subclinical ketosis, but a significant relation between animal age, number of parturition, mastitis and subclinical ketosis was noticed (This result is in accordance with Dohoo et al, 1984). Some investigators explained that post parturient cows have higher susceptibility to local and systemic infectious diseases (1), that may be due to impairment of the respiratory burst of neutrophils which occurs with elevated levels of betahydroxy butyric acid (BHBA). Authors suggest that subclinical ketosis could susceptible cows to infectious diseases (mastitis) and vice versa. Although, we believe that the higher milk yield cows have higher susceptibility to subclinical ketosis, but this study could not confirm this fact.
\end{abstract}

\section{References:}

1. Radostits, O. M. et al (2000): Veterinary Medicine. A Textbook of the Diseases of Cattle, Sheep, Pigs, Goats an Horses. W.B. Saunders. PP:1452-1462.

2. Dohoo, I. R. et al (1984): Disease production and culling in Holstein - Friesian cow. II age, season and sire effect. Prev Vet Med, (2) 665-671. 